\section{The effectiveness of citric acid as an adjunct to surgical re- attachment procedures in humans}

\author{
Smith BA, Mason WE, Morrison EC and Caffesse RG: The effectiveness of citric \\ acid as an adjunct to surgical re-attachment procedures in humans $J$ Clin Periodon- \\ tol 1986; 13: 701-708.
}

\begin{abstract}
The purpose of this study was to clinically evaluate the effect of citric acid on re-attachment and re-adaptation in conjunction with periodontal surgery. A split-mouth design was used in 10 patients involving 30 quadrants of surgery. A total of 120 teeth were treated. Each quadrant consisted of at least 2 teeth from cuspid to second molar. One quadrant was treated with a modified Widman flap alone while the root surfaces of the contralateral side were also treated with a 3 min application of citric acid.

Immediately following hygienic phase and at 3 and 6 months postsurgically, the following measurements were taken in this sequence: gingival index, gingival crevicular fluid flow, plaque index, furcation involvement, level of attachment and probing depth. All the data were statistically analyzed using the paired $t$-test, $\chi^{2}$ and Fisher exact probability test.

The biometric results showed that both surgical techniques resulted in a loss of attachment in shallow pockets and a gain in deeper pockets. Both techniques resulted in similar amounts of recession and probing depth reduction at 6 months. Gingival index, plaque index and furcation values decreased at 3 and 6 months postsurgically. The gingival crevicular fluid values remained essentially the same up to 6 months postsurgically.
\end{abstract}

Billy A. Smith, William E. Mason, Edith C. Morrison and Raul G. Caffesse

Department of Periodontics, The University of Michigan School of Dentistry, Ann Arbor, Michigan, USA

Key words: Periodontal therapy - citric acid - gingivitis - probing depth - attachment level.

Accepted for publication 16 August 1985
The ultimate goal of periodontal therapy is regeneration of an organized, functional fibrous attachment to previously diseased root surfaces.

In the last 10 years, animal and human studies involving demineralization of planed root surfaces have reported clinical and histological evidence of cementogenesis and new connective tissue attachment (Albair et al. 1982, Bogel et al. 1981, Cole et al. 1980, Crigger et al. 1978, Nilveus \& Egelberg 1980, Nilveus et al. 1980, Register \& Burdick 1975). The purpose of this study was to clinically evaluate the effects of citric acid in conjunction with periodontal surgery for new attachment in humans.

\section{Material and Methods}

10 patients, all in good systemic health and diagnosed as having moderate to severe periodontitis, participated in this study. An effort was made to select patients with bilaterally similar bony defects. The group was composed of 6 males and 4 females with an average age of 44.3 and a range from 27 to 56 years. Scaling and root planing were performed together with other hygienic phase procedures (oral hygiene instruction, removal of overhangs, etc.). At the time of re-evaluation at 4 weeks after root instrumentation, patients requiring at least 2 contralateral areas of surgery for new attachment signed a consent form and participated in the study. Each area requiring surgery contained a minimum of 2 teeth from second molar to cuspid. 5 of the 10 patients, 2 males and 3 females, required 4 quadrants of surgery. A total of 30 quadrants of surgery were completed involving 120 teeth. They represented 17 cuspids, 45 bicuspids and 52 molars. Pocket depth measurements ranged from $1-13 \mathrm{~mm}$. There were 326 pockets in the $1-3 \mathrm{~mm}$ range and 274 in the $\geq 4 \mathrm{~mm}$ range at baseline.

Biometric measurements were taken immediately prior to surgery (baseline), and 3 and 6 months post-surgically.
Calibration tests for attachment values were performed prior to the study. The single examiner had a $91.1 \%$ reproducability $\pm 1 \mathrm{~mm}$ for attachment values prior to the start of this study.

The indices and measurements were recorded in the following sequence.

(1) Plaque index (Silness \& Loe 1964) recorded on the 4 surfaces of the involved teeth.

(2) Gingival crevicular fluid flow, using the Periotron 6000, with the measurements taken on the midbuccal and mesial-buccal aspects of two adjacent teeth in each quadrant requiring surgery.

(3) Gingival index (Loe \& Silness 1963) recording the 4 surfaces of all teeth to be treated.

(4) Furcation involvement using a Cattoni probe* with the cementoenamel junction as a fixed point of reference. The measurements for

* UT3-UT4, Hu-Friedy Manufacturing Company, Chigaco, Illinois 60618. 
furcation involvement were taken from the buccal for maxillary and mandibular buccal furcas, from the lingual for mandibular lingual furcas and from the palatal for mesiolingual and disto-lingual furcas.

(5) Clinical attachment levels and probing depths.

Measurements for clinical attachment levels and probing depths were obtained with a calibrated periodontal probe according to the PDI (Ramfjord 1967). These measurements were taken around all teeth to be treated at the following points: (a) midline buccal and lingual and (b) disto-buccal, mesio-buccal and mesio-lingual interproximal line angles as close to the contact as possible with the tip of the probe following the long axis of the tooth.

The treatment and control quadrants were randomly selected at the time of surgery. Surgery was completed as soon as possible following baseline recordings. The surgery involved the elevation of a modified Widman flap (Ramfjord et al. 1975) and the thorough root planing of the exposed surfaces in an attempt to remove all cementum.

The citric acid to be used was supplied in an anhydrous form and was mixed with continuous stirring into room temperature distilled water until a saturated solution with a $\mathrm{pH}$ of 1.0 was obtained. During application of citric acid the exposed surfaces of the flaps were protected with saline moistened gauze. Strips of sterile amalgam squeeze cloth were cut to wrap approximately once around each root. These were saturated with the citric acid solution and left in place for $1 \frac{1}{2}$ minutes, then re- placed with a new strip for the remaining $1 \frac{1}{2}$ minutes. Following the treatment, the amalgam squeeze cloth was removed and the citric acid thoroughly rinsed from the root surfaces with sterile saline. The contralateral control sides were treated with saline for $3 \mathrm{~min}$. Figure eight sutures with 0000 silk were used interpoximally and direct sutures were used in wedge areas to effect closure of the flaps. A periodontal dressing was placed in both test and control quadrants. One week postsurgically, the dressing and sutures were removed and the quadrants were polished using a fluoridated prophylaxis paste. Oral hygiene reinstruction was carried out in detail. Additional appointments at 2, 4 and 6 weeks post-surgically, involved polishing, flossing and oral hygiene instruction. The next appointment was the first 3-month recall.

The data obtained were analyzed using the Michigan Interactive Data Analysis System (MIDAS). The paired $t$-test, $\chi^{2}$ and Fisher exact probability test were used to compare the 2 surgical techniques employed at baseline, 3 and 6 months. Additionally, each technique was tested separately comparing baseline with 3 and 6 months and 3 months with 6 months.

The difference between mean measurements within a subject at 3 and 6 months for $1-3 \mathrm{~mm}$ and $\geq 4 \mathrm{~mm}$ pockets were the variables analyzed. Each patient represented a statistical unit relative to attachment, pocket depth and recession values. Each tooth surface was considered a statistical unit for gingival crevicular fluid flow, plaque index, gingival index, and furcation involve- ment. The null hypothesis was rejected at the 0.05 level of significance.

\section{Results \\ Plaque index}

The plaque index and gingival index were initially analyzed according to the individual surfaces. However, since no significant differences were found between interproximal surfaces and buccal and lingual surfaces, the interproximals and the buccal and lingual surfaces were combined.

The combined buccal and lingual plaque index values remained near baseline values up to 6 months (Table 1). However, there was a slight increase in the $\%$ of 0 scores from 3 months $(46.9 \%)$ to 6 months $(56.7 \%)$. The comparison of citric acid and non-citric acid treated teeth revealed no statistically significant differences.

The combined mesial and distal plaque index values revealed an increase in plaque index $=0$ from $28.8 \%$ at baseline to $38.6 \%$ at 3 months. This \% was maintained with $37.4 \%$ plaque index $=$ 0 at 6 months. The $\%$ of plaque index $=$ 2 and 3 remained very similar from baseline to 6 months. The comparison of citric acid and non-citric acid treated teeth at each interval for all plaque index values exhibited no statistically significant differences.

\section{Gingival index}

The results for combined buccal and lingual gingival index values showed a gradual increase in the percentage of gingival index $=0$ and decrease in $\%$

Table 1. \% distribution of plaque index values at different observation periods for citric acid and non-citric acid treated sites

\begin{tabular}{|c|c|c|c|c|c|c|c|c|c|c|c|c|c|}
\hline \multirow[b]{3}{*}{ Plaque index } & \multirow{3}{*}{$\begin{array}{l}\text { Surgical } \\
\text { technique }\end{array}$} & \multicolumn{6}{|c|}{ Buccal and lingual } & \multicolumn{6}{|c|}{ Mesial and distal } \\
\hline & & \multicolumn{2}{|c|}{ baseline } & \multicolumn{2}{|c|}{3 months } & \multicolumn{2}{|c|}{6 months } & \multicolumn{2}{|c|}{ baseline } & \multicolumn{2}{|c|}{3 months } & \multicolumn{2}{|c|}{6 months } \\
\hline & & $n$ & $(\%)$ & $n$ & $(\%)$ & $n$ & $(\%)$ & $n$ & $(\%)$ & $n$ & $(\%)$ & $n$ & $(\%)$ \\
\hline \multirow[t]{3}{*}{0} & ciitric acid & 54 & 47.4 & 52 & 48.6 & 67 & 55.8 & 30 & 26.3 & 46 & 41.1 & 48 & 40.0 \\
\hline & no citric acid & 55 & 49.1 & 55 & 45.5 & 68 & 57.6 & 35 & 31.3 & 42 & 36.2 & 41 & 34.7 \\
\hline & $\%$ total & & 48.2 & & 46.9 & & 56.7 & & 28.8 & & 38.6 & . & 37.4 \\
\hline \multirow[t]{3}{*}{1} & citric acid & 49 & 43.0 & 39 & 36.4 & 38 & 31.7 & 56 & 49.1 & 36 & 32.1 & 51 & 42.5 \\
\hline & no citric acid & 50 & 44.6 & 53 & 43.8 & 42 & 35.6 & 46 & 41.1 & 41 & 35.3 & 49 & 41.5 \\
\hline & $\%$ total & & 43.8 & & 40.4 & & 33.6 & & 45.1 & & 33.8 & & 42.0 \\
\hline \multirow[t]{6}{*}{$2+3$} & citric acid & 11 & 9.6 & 16 & 15.0 & 15 & 12.5 & 28 & 24.6 & 30 & 26.8 & 21 & 17.5 \\
\hline & no citric acid & 7 & 6.3 & 13 & 10.7 & 8 & 6.8 & 31 & 27.7 & 33 & 28.4 & 28 & 23.7 \\
\hline & $\%$ total & & 8.0 & & 12.7 & & 9.7 & & 26.1 & & 27.6 & & 20.6 \\
\hline & $n$ total & 226 & & 228 & & 238 & & 226 & & 228 & & 238 & \\
\hline & $\chi^{2}$ & \multicolumn{2}{|c|}{0.891} & \multicolumn{2}{|c|}{1.672} & \multicolumn{2}{|c|}{2.321} & \multicolumn{2}{|c|}{1.500} & \multicolumn{2}{|c|}{0.579} & \multicolumn{2}{|c|}{1.574} \\
\hline & ${ }^{*} p$ & \multicolumn{2}{|c|}{0.6407} & \multicolumn{2}{|c|}{0.4335} & \multicolumn{2}{|c|}{0.3133} & \multicolumn{2}{|c|}{0.4724} & \multicolumn{2}{|c|}{0.7485} & \multicolumn{2}{|c|}{0.4522} \\
\hline
\end{tabular}

$\chi^{2}$ test. 
of gingival index $=1$ from baseline to 6 months (Table 2). The \% of gingival index $=2$ and 3 remained essentially constant at all time periods. When comparing citric acid and non-citric acid treated teeth at each interval, no statistically significant differences were evident.

The combined mesial and distal results showed about half of the values to be 0 and 1 and half 2 and 3 at each time interval. The comparison of citric acid and non-citric acid treated teeth revealed no statistically significant differ- ences. The gingival index 0 and 1 values were combined for buccal and lingual at baseline and mesial and distal at 3 and 6 months due to insufficient frequency distribution for a valid $\chi^{2}$ test.

\section{Gingival crevicular fluid}

The gingival crevicular fluid measurements ranged from 0 to 48 . They were grouped into two categories: $0-10$ and 11-48 (Table 3).

The majority of mesial buccal measurements were between 0 and 10 at baseline, 3 months and 6 months. There were differences between crevicular fluid values for citric acid and non-citric acid treated teeth, but none were statistically significant.

$94 \%$ of the buccal baseline gingival crevicular fluid measurements were between 0 and 10 . At 3 months there was a slight shift to the 11 to 48 range, which continued at 6 months. The differences between citric acid and non-citric acid treated teeth were not statistically significant.

Table 2. \% distribution of gingival index values at different observation periods for citric acid and non-citric acid treated sites

\begin{tabular}{|c|c|c|c|c|c|c|c|c|c|c|c|c|c|}
\hline \multirow{3}{*}{$\begin{array}{l}\text { Gingival } \\
\text { index }\end{array}$} & \multirow{3}{*}{$\begin{array}{l}\text { Surgical } \\
\text { technique }\end{array}$} & \multicolumn{6}{|c|}{ Buccal and lingual } & \multicolumn{6}{|c|}{ Mesial and distal } \\
\hline & & \multicolumn{2}{|c|}{ baseline } & \multicolumn{2}{|c|}{3 months } & \multicolumn{2}{|c|}{6 months } & \multicolumn{2}{|c|}{ baseline } & \multicolumn{2}{|c|}{3 months } & \multicolumn{2}{|c|}{6 months } \\
\hline & & $n$ & $(\%)$ & $n$ & $(\%)$ & $n$ & $(\%)$ & $n$ & $(\%)$ & $n$ & $(\%)$ & & $(\%)$ \\
\hline \multirow[t]{3}{*}{$\overline{0+1}$} & citric acid & 83 & 73.5 & 84 & 76.4 & 94 & 78.3 & 56 & 49.1 & 44 & 40.0 & 53 & 44.2 \\
\hline & no citric acid & 84 & 74.3 & 83 & 70.3 & 85 & 72.0 & 49 & 43.8 & 54 & 45.8 & 65 & 55.1 \\
\hline & $\%$ total & & 73.9 & & 73.3 & & 75.2 & & 46.5 & & 43.0 & & 49.6 \\
\hline \multirow[t]{7}{*}{$2+3$} & citric acid & 30 & 26.5 & 26 & 23.6 & 26 & 21.7 & 58 & 50.9 & 66 & 60.0 & 67 & 55.8 \\
\hline & no citric acid & 29 & 25.7 & 35 & 29.7 & 33 & 28.0 & 63 & 56.3 & 64 & 54.2 & 53 & 44.9 \\
\hline & $\%$ total & & 26.1 & & 26.7 & & 24.8 & & 53.5 & & 57.0 & & 50.4 \\
\hline & & \multirow{2}{*}{\multicolumn{2}{|c|}{226}} & \multirow{2}{*}{\multicolumn{2}{|c|}{228}} & \multirow{2}{*}{\multicolumn{2}{|c|}{238}} & \multirow{2}{*}{\multicolumn{2}{|c|}{226}} & \multirow{2}{*}{\multicolumn{2}{|c|}{228}} & & \\
\hline & $n$ total & & & & & & & & & & & & \\
\hline & $\chi^{2}$ & \multicolumn{2}{|c|}{0.00} & \multicolumn{2}{|c|}{4.175} & \multicolumn{2}{|c|}{1.333} & \multicolumn{2}{|c|}{0.457} & \multicolumn{2}{|c|}{0.554} & \multicolumn{2}{|c|}{2.417} \\
\hline & $p$ & \multicolumn{2}{|c|}{$0.5000 *$} & \multicolumn{2}{|c|}{$0.1240^{* *}$} & \multicolumn{2}{|c|}{$0.5135^{* *}$} & \multicolumn{2}{|c|}{$0.2495^{*}$} & \multicolumn{2}{|c|}{$0.2284 *$} & \multicolumn{2}{|c|}{$0.0599 *$} \\
\hline
\end{tabular}

* Fisher exact probability test.

** $\chi^{2}$ test.

Table 3. \% distribution of gingival crevicular fluid flow values at different observation periods for citric acid and non-citric acid treated sites

\begin{tabular}{|c|c|c|c|c|c|c|c|c|c|c|c|c|c|}
\hline \multirow{2}{*}{$\begin{array}{l}\text { Gingival } \\
\text { crevicular } \\
\text { fluid flow }\end{array}$} & \multirow{2}{*}{$\begin{array}{l}\text { Surgical } \\
\text { technique }\end{array}$} & \multicolumn{6}{|c|}{ Mesiobuccal } & \multicolumn{2}{|c|}{ baseline } & \multicolumn{2}{|c|}{$\begin{array}{l}\text { Buccal } \\
3 \text { months }\end{array}$} & \multicolumn{2}{|c|}{6 months } \\
\hline & & $n$ & $(\%)$ & $n$ & $(\%)$ & $n$ & $(\%)$ & $n$ & $(\%)$ & $n$ & $(\%)$ & $n$ & $(\%)$ \\
\hline \multirow[t]{3}{*}{$0-10$} & citric acid & 23 & 92.0 & 18 & 75.0 & 21 & 77.8 & 23 & 92.0 & 21 & 87.5 & 20 & 74.1 \\
\hline & no citric acid & 21 & 84.0 & 23 & 88.5 & 23 & 85.2 & 24 & 96.0 & 23 & 88.5 & 22 & 81.5 \\
\hline & $\%$ total & & 88.0 & & 82.0 & & 82.0 & & 94.0 & & 88.0 & & 78.0 \\
\hline \multirow[t]{5}{*}{$11-48$} & citric & 2 & 8.0 & 6 & 25.0 & 6 & 22.2 & 2 & 8.0 & 3 & 12.5 & 7 & 25.9 \\
\hline & no citric acid & 4 & 16.0 & 3 & 11.5 & 4 & 14.8 & 1 & 4.0 & 3 & 11.5 & 5 & 18.5 \\
\hline & $\%$ total & & 12.0 & & 18.0 & & 18.0 & & 6.0 & & 12.0 & & 22.0 \\
\hline & $n$ total & \multicolumn{2}{|c|}{50} & \multicolumn{2}{|c|}{50} & \multicolumn{2}{|c|}{54} & \multicolumn{2}{|c|}{50} & \multicolumn{2}{|c|}{50} & \multicolumn{2}{|c|}{54} \\
\hline & $p^{*}$ & \multicolumn{2}{|c|}{0.33355} & \multicolumn{2}{|c|}{0.192726} & \multicolumn{2}{|c|}{0.363829} & \multicolumn{2}{|c|}{0.50000} & \multicolumn{2}{|c|}{0.626502} & \multicolumn{2}{|c|}{0.372274} \\
\hline
\end{tabular}

* Fisher exact probability test.

Table 4. Attachment changes from baseline to 3 months and 6 months in 1-3 mm pockets for citric and non-citric acid treated sites

\begin{tabular}{|c|c|c|c|c|c|c|c|c|c|c|c|}
\hline \multirow[b]{2}{*}{ Surgical technique } & \multirow{2}{*}{$\begin{array}{c}\text { Baseline } \\
n=10 \\
\text { mean } \\
(\mathrm{mm})\end{array}$} & \multicolumn{5}{|c|}{$\begin{array}{c}3 \text { months } \\
n=10\end{array}$} & \multicolumn{5}{|c|}{$\begin{array}{c}6 \text { months } \\
n=10\end{array}$} \\
\hline & & $\begin{array}{l}\text { mean } \\
(\mathrm{mm})\end{array}$ & $\begin{array}{l}\text { mean } \\
\text { diff. } \\
(\mathrm{mm})\end{array}$ & S.D. & $t$ stat.* & $p^{*}$ & $\begin{array}{l}\text { mean } \\
(\mathrm{mm})\end{array}$ & $\begin{array}{l}\text { mean } \\
\text { diff. } \\
(\mathrm{mm})\end{array}$ & S.D. & $t$. stat.* & $p^{*}$ \\
\hline Citric acid & 3.06 & 3.27 & 0.21 & 0.69700 & -0.95191 & 0.3660 & 3.37 & -0.31 & 0.37218 & -2.6341 & 0.0272 \\
\hline $\begin{array}{l}\text { citric acid versus no } \\
\text { acid }\end{array}$ & & & -0.23 & 0.77499 & -0.94108 & 0.3712 & & 0.06 & 0.69726 & 0.29965 & 0.7712 \\
\hline no citric acid & 2.79 & 3.23 & -0.44 & 0.72604 & -1.9184 & 0.0873 & 3.04 & -0.25 & 0.53476 & -1.4426 & 0.1830 \\
\hline
\end{tabular}

* paired $t$-test. 
Attachment level (all surfaces included)

The results showed that in 1-3 mm pockets at 3 and 6 months, both citric acid and non-citric acid treated teeth lost attachment (Table 4). The loss of attachment for citric acid treated teeth was statistically significant at 6 months.

In $\geq 4 \mathrm{~mm}$ pockets, both citric acid and non-citric acid treated teeth gained attachment at 3 and 6 months (Table 5). None of these results were statistically significant.

\section{Interproximal attachment}

At 3 months in 1-3 mm interproximal pockets, both citric acid and non-citric acid treated teeth, lost attachment (Table 6). The citric acid treated teeth lost $0.49 \mathrm{~mm}$ of attachment which was significant. The difference between treatments was not significant. The interproximal attachment change from baseline to 6 months in $1-3 \mathrm{~mm}$ pockets showed a similar trend. None of the results were of statistical significance.

In interproximal pockets $\geq 4 \mathrm{~mm}$, both citric acid and non-citric acid treated teeth gained attachment at 3 months, but none of the changes were significant (Table 7). At 7 months, non-citric acid as well as citric treated teeth, maintained the gain in attachment interproximally, but neither gain was significant.

\section{Probing depth}

At 3 months in $1-3 \mathrm{~mm}$ pockets, the non-citric acid treated teeth showed a slight increase in probing depth, while the citric acid treated teeth had a statistically significant decrease in probing depth (Table 8). The difference between the 2 treatments was $0.48 \mathrm{~mm}$, which was significant. At 6 months in 1-3 mm pockets, both treatments resulted in statistically insignificant reductions in probing depth.

In $\geq 4 \mathrm{~mm}$ pockets, both citric acid and non-citric acid treated teeth exhibited pocket reduction with values that were highly significant (Table 9). Citric acid treated teeth showed 1.95

Table 5. Attachment changes from baseline to 3 months and 6 months $\geq 4 \mathrm{~mm}$ pockets for citric acid and non-citric acid treated sites

\begin{tabular}{|c|c|c|c|c|c|c|c|c|c|c|c|}
\hline . & $\begin{array}{c}\text { Baseline } \\
n=10\end{array}$ & & & $\begin{array}{c}3 \text { months } \\
n=10\end{array}$ & & & & & $\begin{array}{c}6 \text { months } \\
n=10\end{array}$ & 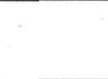 & \\
\hline Surgical technique & $\begin{array}{l}\text { mean } \\
(\mathrm{mm})\end{array}$ & $\begin{array}{l}\text { mean } \\
(\mathrm{mm})\end{array}$ & $\begin{array}{l}\text { mean } \\
\text { diff. } \\
(\mathrm{mm})\end{array}$ & S.D & $t$. stat.* & $p^{*}$ & $\begin{array}{l}\text { mean } \\
(\mathrm{mm})\end{array}$ & $\begin{array}{l}\text { mean } \\
\text { diff. } \\
(\mathrm{mm})\end{array}$ & S.D. & $t$. stat.* & $p^{*}$ \\
\hline citric acid & 4.32 & 0.30 & 0.89173 & 1.0707 & 0.3122 & 4.08 & 0.24 & 0.9368 & 0.8055 & 0.4413 & \\
\hline $\begin{array}{l}\text { citric acid versus no citric } \\
\text { acid }\end{array}$ & & & -0.10 & 0.96522 & -0.32849 & 0.7501 & & 0.06 & 0.67868 & 0.2788 & 0.7867 \\
\hline no citric acid & 4.24 & 4.04 & 0.20 & 0.41268 & 1.5452 & 0.1567 & 3.94 & 0.30 & 0.64647 & 1.46 & 0.1783 \\
\hline
\end{tabular}

* paired $t$-test.

Table 6. Interproximal attachment changes from baseline to 3 months and 6 months in $1-3 \mathrm{~mm}$ pockets for citric acid and non-citric acid treated sites

\begin{tabular}{|c|c|c|c|c|c|c|c|c|c|c|c|}
\hline & $\begin{array}{c}\text { Baseline } \\
n=10\end{array}$ & & & $\begin{array}{c}3 \text { month } \\
n=10\end{array}$ & & & & & $\begin{array}{c}6 \text { months } \\
n=10\end{array}$ & & \\
\hline Surgical technique & $\begin{array}{l}\text { mean } \\
(\mathrm{mm})\end{array}$ & $\begin{array}{l}\text { mean } \\
(\mathrm{mm})\end{array}$ & $\begin{array}{c}\text { mean } \\
\text { diff. } \\
(\mathrm{mm})\end{array}$ & S.D. & $t$. stat.* & $p^{*}$ & $\begin{array}{l}\text { mean } \\
(\mathrm{mm})\end{array}$ & $\begin{array}{l}\text { mean } \\
\text { diff. } \\
(\mathrm{mm})\end{array}$ & S.D. & $t$. stat.* & $p^{*}$ \\
\hline citric acid & 3.09 & 3.58 & -0.49 & 0.62546 & -2.3433 & 0.0472 & 3.48 & -0.39 & 0.54396 & -2.1336 & 0.0654 \\
\hline $\begin{array}{l}\text { citric acid versus } n \\
\text { acid }\end{array}$ & & & -0.04 & 1.0961 & -0.17993 & 0.8617 & & -0.05 & 0.96528 & 0.038631 & 0.9701 \\
\hline no citric acid & 2.59 & 3.04 & -0.45 & 1.1046 & -1.2850 & 0.2309 & 2.93 & -0.34 & 0.73142 & -1.4570 & 0.1791 \\
\hline
\end{tabular}

* paired $t$-test.

Table 7. Interproximal attachment changes from baseline to 3 months and 6 months in $\geq 4$ mm pockets for citric and non-citric acid treated sites

\begin{tabular}{|c|c|c|c|c|c|c|c|c|c|c|c|}
\hline & $\begin{array}{c}\text { Baseline } \\
n=10\end{array}$ & & & $\begin{array}{l}3 \text { month } \\
n=10\end{array}$ & & & & & $\begin{array}{l}6 \text { month } \\
n=10\end{array}$ & & \\
\hline Surgical technique & $\begin{array}{l}\text { mean } \\
(\mathrm{mm})\end{array}$ & $\begin{array}{l}\text { mean } \\
(\mathrm{mm})\end{array}$ & $\begin{array}{l}\text { mean } \\
\text { diff. } \\
(\mathrm{mm})\end{array}$ & S.D. & t. stat.* & $p^{*}$ & $\begin{array}{l}\text { mean } \\
(\mathrm{mm})\end{array}$ & $\begin{array}{c}\text { mean } \\
\text { diff. } \\
(\mathrm{mm})\end{array}$ & S.D. & $t$. stat.* & $p^{*}$ \\
\hline citric acid & 4.08 & 3.91 & 0.17 & 0.75902 & 0.72757 & 0.4854 & 3.96 & 0.12 & 0.77649 & 0.52508 & 0.6122 \\
\hline $\begin{array}{l}\text { citric acid versus no citric } \\
\text { acid }\end{array}$ & & & -0.03 & 0.84933 & -0.14608 & 0.8871 & & 0.08 & 0.63596 & 0.35326 & 0.7320 \\
\hline no citric acid & 4.13 & 3.99 & 0.14 & 0.4005 & 1.0691 & 0.3129 & 3.93 & 0.20 & 0.73310 & 0.86260 & 0.4108 \\
\hline
\end{tabular}

\footnotetext{
* Paired $t$-test.
} 
Table 8. Probing depth changes from baseline to 3 months and 6 months in 1-3 mm pockets for citric acid and non-citric acid treated sites

\begin{tabular}{|c|c|c|c|c|c|c|c|c|c|c|c|}
\hline & $\begin{array}{c}\text { Baseline } \\
n=10\end{array}$ & & & $\begin{array}{c}3 \text { month } \\
n=10\end{array}$ & & & & & $\begin{array}{c}6 \text { month } \\
n=10\end{array}$ & & \\
\hline Surgical technique & $\begin{array}{l}\text { mean } \\
(\mathrm{mm})\end{array}$ & $\begin{array}{l}\text { mean } \\
(\mathrm{mm})\end{array}$ & $\begin{array}{l}\text { mean } \\
\text { diff. } \\
(\mathrm{mm})\end{array}$ & S.D. & $t$. stat.* & $p^{*}$ & $\begin{array}{l}\text { mean } \\
(\mathrm{mm})\end{array}$ & $\begin{array}{l}\text { mean } \\
\text { diff. } \\
(\mathrm{mm})\end{array}$ & S.D. & t. stat.* & $p^{*}$ \\
\hline citric acid & 2.48 & 2.05 & 0.43 & 0.31839 & 4.3442 & 0.0019 & 2.34 & 0.14 & 0.3163 & 1.4514 & 0.1806 \\
\hline $\begin{array}{l}\text { citric acid versus no } \\
\text { acid }\end{array}$ & & & -0.48 & 0.67689 & -2.2578 & 0.0504 & & -0.01 & 0.39741 & -0.070883 & 0.9450 \\
\hline no citric acid & 2.39 & 2.44 & -0.05 & 0.68647 & -0.21146 & 0.8372 & 2.26 & 0.13 & 0.43569 & 0.98905 & 0.3485 \\
\hline
\end{tabular}

* Paired $t$-test.

Table 9. Probing depth changes from baseline to 3 months and 6 months in $\geq 4 \mathrm{~mm}$ pockets for citric acid and non-citric acid treated sites

\begin{tabular}{|c|c|c|c|c|c|c|c|c|c|c|c|}
\hline \multirow[b]{2}{*}{ Surgical technique } & \multirow{2}{*}{$\begin{array}{c}\begin{array}{c}\text { Baseline } \\
n=10\end{array} \\
\text { mean } \\
(\mathrm{mm})\end{array}$} & \multicolumn{5}{|c|}{$\begin{array}{c}3 \text { months } \\
n=10\end{array}$} & \multicolumn{5}{|c|}{$\begin{array}{c}6 \text { months } \\
n=10\end{array}$} \\
\hline & & $\begin{array}{l}\text { mean } \\
(\mathrm{mm})\end{array}$ & $\begin{array}{l}\text { mean } \\
\text { diff. } \\
(\mathrm{mm})\end{array}$ & S.D. & t. stat.* & $p^{*}$ & $\begin{array}{l}\text { mean } \\
(\mathrm{mm})\end{array}$ & $\begin{array}{l}\text { mean } \\
\text { diff. } \\
(\mathrm{mm})\end{array}$ & S.D. & t. stat.* & $p^{*}$ \\
\hline citric acid & 4.82 & 2.87 & 1.95 & 0.74144 & 8.2998 & 0.0001 & 3.34 & 1.48 & 0.66574 & 7.0092 & 0.0001 \\
\hline citric acid versus no citric & & & -0.39 & 0.81408 & -1.5179 & 0.1634 & & -0.07 & 0.38921 & 0.58069 & 0.5757 \\
\hline no citric acid & 4.99 & 3.43 & 1.56 & 0.57974 & 8.4833 & 0.0001 & 3.44 & 1.55 & 0.62099 & 7.8792 & 0.0001 \\
\hline
\end{tabular}

* Paired $t$-test.

Table 10. Recession values from baseline to 3 months and 6 months for citric acid and non-citric acid treated sites

\begin{tabular}{|c|c|c|c|c|c|c|c|c|c|c|}
\hline & & & $\begin{array}{c}3 \text { months } \\
n=10\end{array}$ & $1-3 n$ & n pockets & & & $\begin{array}{c}6 \text { months } \\
n=10\end{array}$ & & \\
\hline $\begin{array}{l}\text { Surgical } \\
\text { technique }\end{array}$ & $\begin{array}{c}\text { recession } \\
\text { from } \\
\text { baseline } \\
(\mathrm{mm})\end{array}$ & $\begin{array}{l}\text { mean } \\
\text { diff. } \\
(\mathrm{mm})\end{array}$ & S.D. & t. sta.* & $p^{*}$ & $\begin{array}{c}\text { recession } \\
\text { from } \\
\text { baseline } \\
(\mathrm{mm})\end{array}$ & $\begin{array}{c}\text { mean } \\
\text { diff. } \\
(\mathrm{mm})\end{array}$ & S.D. & t. stat.* & $p^{*}$ \\
\hline $\begin{array}{l}\text { citric acid } \\
\text { no citric acid }\end{array}$ & $\begin{array}{l}0.55 \\
0.51\end{array}$ & -0.04 & 0.75074 & -0.1735 & 0.8661 & $\begin{array}{l}0.42 \\
0.42 \\
\end{array}$ & 0.00 & 0.75074 & -0.17350 & 0.8661 \\
\hline \multicolumn{11}{|c|}{$\geq 4 \mathrm{~mm}$ pockets } \\
\hline $\begin{array}{l}\text { citric acid } \\
\text { no citric acid }\end{array}$ & $\begin{array}{l}1.64 \\
1.35\end{array}$ & -0.29 & 0.66772 & -1.3758 & 0.2022 & $\begin{array}{l}1.24 \\
1.25\end{array}$ & 0.01 & 0.011635 & 0.51142 & 0.9442 \\
\hline
\end{tabular}

* Paired $t$-test.

mm reduction in probing depth and non-citric acid treated teeth $1.56 \mathrm{~mm}$ reduction at 3 months. The difference between treatments was not significant. At 6 months, both non-citric acid and citric acid treated teeth maintained reductions in probing depth, which were statistically significant. The difference between treatments was minimal and not statistically significant.

\section{Recession}

Recession from baseline to 3 months in 1-3 mm pockets was $0.51 \mathrm{~mm}$ for non- citric acid treated and $0.55 \mathrm{~mm}$ for citric acid treated teeth (Table 10). In $\geq 4 \mathrm{~mm}$ pockets, non-citric acid treated teeth exhibited $1.35 \mathrm{~mm}$ recession while citric acid treated teeth had $1.64 \mathrm{~mm}$. There were no significant differences between the citric acid and non-citric acid treated teeth. The reduced degree of recession from baseline to 6 months showed that there was some rebound of the gingival tissues. Citric acid and non-citric acid treated teeth had $0.42 \mathrm{~mm}$ of recession in $1-3 \mathrm{~mm}$ pockets. In $\geq 4 \mathrm{~mm}$ pockets, citric acid treated teeth had $1.24 \mathrm{~mm}$ of recession while non-citric acid treated teeth had $1.25 \mathrm{~mm}$ of recession. The differences were not statistically significant.

\section{Furcations}

The depth of the involvement of furcations was stratified into two groups: 1-3 $\mathrm{mm}$ and $\geq 4 \mathrm{~mm}$.

The results for the maxillary buccal, mesial and distal furcations and mandibular buccal and lingual furcations were analyzed individually. No significant differences were found between surfaces or between arches, therefore, 
Table 11. \% distribution of furcations according to probing depth at different observation periods for citric acid and non-citric acid treated sites

\begin{tabular}{|c|c|c|c|c|c|c|c|c|c|c|c|c|}
\hline \multirow[b]{3}{*}{ Surgical technique } & \multicolumn{4}{|c|}{ Baseline } & \multicolumn{4}{|c|}{3 months } & \multicolumn{4}{|c|}{6 months } \\
\hline & \multicolumn{2}{|c|}{$1-3 \mathrm{~mm}$} & \multicolumn{2}{|c|}{$>4 \mathrm{~mm}$} & \multicolumn{2}{|c|}{$1-3 \mathrm{~mm}$} & \multicolumn{2}{|c|}{$>4 \mathrm{~mm}$} & \multicolumn{2}{|c|}{$1-3 \mathrm{~mm}$} & \multicolumn{2}{|c|}{$>4 \mathrm{~mm}$} \\
\hline & $n$ & $(\%)$ & $n$ & $(\%)$ & $n$ & $(\%)$ & $n$ & $(\%)$ & $n$ & $(\%)$ & $n$ & $(\%)$ \\
\hline citric acid & 25 & 39.7 & 38 & 60.3 & 37 & 63.8 & 21 & 36.2 & 39 & 60.9 & 25 & 39.1 \\
\hline no citric acid & 27 & 47.4 & 30 & 52.6 & 44 & 71.0 & 18 & 29.0 & 45 & 72.6 & 17 & 27.4 \\
\hline$\%$ total & & 43.55 & & 56.45 & & 67.4 & & 32.6 & & 66.75 & & 33.25 \\
\hline$p^{*}$ & & 0.2534 & & & & 0.2599 & & & & 0.1155 & & \\
\hline$n$ & 52 & & 68 & & 81 & $\cdot$ & 39 & & 84 & & 42 & \\
\hline$n$ total & & 120 & & & & 120 & & & & 126 & & \\
\hline
\end{tabular}

* Fisher exact probability test.

all furcations were combined and analyzed as a group (Table 11). The results showed a similar pattern of reduction in probing depth for both treatments, which was most evident at 3 months. From 3 to 6 months, the reductions were maintained in most cases. No statistically significant differences were found between the 2 approaches to treatment.

\section{Discussion}

The results of this study have shown that the use of citric acid as a root conditioner in conjunction with modified Widman flap surgery provided no significant differences in clinical probing attachment levels. Both citric acid and non-citric acid treated quadrants showed comparable clinical results as measured by attachment level, probing depth and recession.

The loss of attachment in 1-3 mm pockets in non-citric acid treated teeth at 3 and 6 months is in agreement with previous studies (Burgett et al. 1977, Knowles et al. 1979, Hill et al. 1981). Even when the interproximals alone were computed, there was loss of attachment in $1-3 \mathrm{~mm}$ pockets. Citric acid treatment did not affect attachment changes in shallow pockets. The deeper pockets, $\geq 4 \mathrm{~mm}$, gained attachment with and without citric acid. No significant difference was evident between citric acid and non-citric acid treated teeth. These results are in disagreement with previous studies using citric acid during surgery (Cole et al. 1980, Renvert \& Egelberg 1981, Stahl \& Froum 1977).

Cole et al. (1980) compared modified Widman flap surgery with and without citric acid application. Pockets were treated in contralateral sites. At 6 months, the pooled acid treated surfaces had a mean gain in probing attachment level of $2.1 \mathrm{~mm}$ as compared to $1.5 \mathrm{~mm}$ for controls. No details of the types of defects treated were available. If intraosseous defects were selected for treatment, then perhaps the gain in attachment may be attributable to their morphology.

Renvert \& Egelberg (1981) showed an average gain in probing attachment level of $2 \mathrm{~mm}$ six months post-surgically with citric acid. Non-citric acid treated teeth gained 1.1-1.2 mm of attachment. However, they only treated selected interproximal intraosseous defects with residual probing depth of $6 \mathrm{~mm}$ or greater. Perhaps deeper pockets offer greater potential for new attachment in conjunction with citric acid application. Also, not all defects in the present study represented intraosseous morphology and therefore, perhaps did not have similar potential for regeneration.

Probing depth changes showed that in shallow $1-3 \mathrm{~mm}$ pockets at 3 months, the citric acid teeth had a highly significant reduction compared to non-citric acid treated teeth. The difference between citric acid and non-citric acid treated teeth was $0.48 \mathrm{~mm}$ which was significant. There is no obvious explanation for the difference, but two possibilities are marginal flap necrosis and/ or accelerated healing with citric acid. Marginal flap necrosis has not been reported as an observation in previous clinical studies and is clinically improbable, since no complications were observed during the post-surgical followup appointments. Cole et al. (1980) reported better soft tissue healing with citric acid treatment as compared to saline treated controls.

Probing depth changes in $\geq 4 \mathrm{~mm}$ pockets were significant, with greater reduction in citric acid treated teeth at 3 months. However, the 3- to 6-month changes may indicate the healing in non-citric acid treated teeth may be delayed compared to citric acid treated teeth, since non-citric acid treated teeth gained clinical attachment from 3 to 6 months in $\geq 4 \mathrm{~mm}$ pockets. Probing depth changes from baseline to 6 months revealed essentially identical reductions in probing depth. However, pocket reduction was greater in citric acid treated teeth the first three months.

Recession values from baseline to three months in 1-3 $\mathrm{mm}$ pockets were similar. Recession values for $\geq 4 \mathrm{~mm}$ pockets showed $0.29 \mathrm{~mm}$ greater recession with citric acid, which may partially explain the changes in probing depth. Stahl \& Froum (1977) found an average recession of $0.7 \mathrm{~mm}$ on the 7 test teeth. However, the authors did not state whether they used modified Widman flaps but did state that they attempted to retain "as much of the gingival margin as possible". Also, recession values were pooled for treatment teeth and controls which did not allow for comparison. Recession values at the end of the present study were essentially identical for citric acid and non-citric acid treated teeth. This reinforced the consistency of the surgical protocal for both treatments.

The plaque index, gingival index and gingival crevicular fluid flow measurements did not show significant differences between techniques at any of the time intervals. The fact that all measurements were taken following the completion of the hygienic phase may explain the overall tendency for low scores. Also, polishing, flossing and oral hygiene instruction were carried out at each of the four postsurgical appointments, therefore, plaque control was continually reinforced. These results are in agreement with several reports indicating that frequent professional care and adequate oral hygiene are effective in the maintenance of gingival health (Nyman et al. 1975, Rosling et al. 1976).

It was expected that plaque index values would remain essentially the same 
from baseline through 6 months. However, a slight increase in the $\%$ of plaque index $=0$ for buccal and linguals from 3 months to 6 months occurred. Further improvement in oral hygiene appears to have taken place following surgery. The mesial and distal plaque index values improved from baseline to 3 months and remained essentially the same at 6 months. Whenever interproximal recession allowed, Prox-A-Brush interdental aids were recommended for use.

Some patients reported postsurgical root sensitivity supragingivally on the citric acid treated side. This may be partly explained by the agressive root planing aimed at total cementum removal and subsequent citric acid application. Also, it was difficult to restrict the area of citric acid application since it is a clear nonviscous fluid which resulted in some of the supragingival root surfaces being exposed to citric acid. The maxillary teeth were especially vulnerable to this due to gravity causing a flow of citric acid coronally. One might have suspected root sensitivity would have affected plaque control, especially at the buccal and lingual surfaces. However, the results did not support this since there were no statistically significant difference in any instance between citric acid and non-citric acid treated teeth. Previous clinical studies have not reported postsurgical root sensitivity.

The results of this clinical study indicate that there is no added advantage in using citric acid during modified Widman flap surgery in humans. Essentially similar attachment gains in deeper pockets and attachment losses in shallower ones can be expected. Recession and pocket reduction are not enhanced or decreased following citric acid application. Finally, no clinical evidence of improved or accelerated healing can be expected.

\section{Conclusions}

Within the limits of this study, the following conclusions can be made:

(1) The use of citric acid with the modified Widman flap does not result in significant additional clinical attachment gain, pocket reduction or change in recession as compared to the modified Widman flap alone.

(2) There was no significant difference between citric acid treated and noncitric acid treated sites with respect to Gingival Index, Plaque Index, gingival crevicular fluid flow, and furcation values.

\section{Zusammenfassung}

Die Wirkung der Zitronensäure als Adjuvans zur chirurgischen Reattachmentbehandlung beim Menschen

In der hier vorliegenden Studie sollte, in Verbindung mit parodontalchirurgischer $\mathrm{Be}$ handlung, der Effekt der Zitronensäure auf das Reattachment und die Readaptation klinisch bewertet werden. Bei 10 Patienten wurde in 30 behandelten Quadranten eine "splitmouth" Formgebung des klinischen Versuches gewählt. Zusammengenommen wurden 120 Zähne behandelt. Jeder Quadrant bestand aus mindestens 2 Zähnen vom Eckzahn bis zum 2. Molaren. Ein Quadrant wurde ausschliesslich mit der modifizierten Widman'schen Lappenoperation behandelt, während die Wurzeloberflächen der kontralateralen Seite zusätzlich 3 Minuten lang mit Zitronensäure behandelt wurden.

Direkt nach der Hygienephase und 3 und 6 Monate nach dem Eingriff wurden Messungen in folgendem Ablauf vorgenommen: Gingival Index, Sulkus-Fluid Fliessrate, Plaque Index, Einbeziehung der Furkation, Attachmentniveau und Sondierungstiefe. Alle Daten wurden statistisch mit dem paarigen $t$ Test, dem $\chi^{2}$ Test und mit dem exakten Wahrscheinlichkeitstest nach Fisher analysiert. Die biometrischen Ergebnisse zeigten, dass beide chirurgischen Techniken in flachen Taschen einen Attachmentverlust verursachten; in tie- fen Taschen wurde ein Attachmentgewinn erreicht. Beide Behandlungstechniken resultierten nach 6 Monaten in ähnlichen Rezessionsgrössen und Reduktionswerten der Taschentiefen. 3 und 6 Monate nach dem chirurgischen Eingriff hatten sich die Werte sowohl des Gingivalindexes als auch des Furkationsbeteiligungsindexes verringert. Die Werte der Sulkus-Fluid Fliessrate blieben nach dem Eingriff im grossen und ganzen unverändert.

\section{Résumé}

Efficacité de l'acide citrique comme adjuvant des méthodes chirurgicales visant à la réattache chez l'homme

Le but de cette étude était l'évaluation de l'effet obtenu sur la réattache et la réadaptation par l'acide citrique employé conjointement à la chirurgie parodontale. L'étude, conçue suivant une méthode "de bouche divisée" (split-mouth) a porté sur 10 patients totalisant 30 quadrants à opérer. Au total, 120 dents ont été traitées. Chaque quadrant se composait d'au moins 2 dents de la canine à la $2^{\text {eme }}$ molaire. Dans un de ces quadrants, une opération à lambeau de Widman modifiée a été utilisée seule, tandis que, dans le côté controlatéral on pratiquait aussi un traitement des surfaces radiculaires à l'acide citrique pendant $3 \mathrm{~min}$.

Les enregistrements suivants ont été pratiqués, dans l'ordre indiqué, immédiatement après la phase hygiénique, puis 3 mois et 6 mois après chirurgie: indice gingival, écoule-

\section{References}

Albair, W. B., Cobb, C. M. \& Killoy, W. J. (1982) Connective tissue attachment to periodontally diseased roots after citric acid demineralization. Journal of Periodontology 53, 515-526.

Bogel, G., Adams, D., Crigger, M., Klinge, P. \& Egelberg, J. (1981) New attachment after surgical treatment and acid conditioning of roots in naturally occurring P.D.D. in dogs. Journal of Periodontal Research 16, 130-133.

Burgett, F. G., Knowles, J. W., Nissle, R. R., Shick, R. A. \& Ramfjord, S. P. (1977) Short term results of three modalities of periodontal treatment. Journal of Periodontology 48, $131-139$.

Cole, R. T., Crigger, M., Bogle, G., Egelberg, J. \& Selvig, K. A. (1980) Connective tissue regeneration to periodontally diseased teeth. A histological study. Journal of Periodontal Research 15, 1-9.

Cole, R. T., Nilveus, I., Ainamo, J., Bogel, G., Crigger, M. \& Egelberg, J. (1980) Effect of citric acid application on healing after modified Widman surgery. Journal of Clinical Periodontology 7, 345 .

Crigger, M., Bogle, G., Nilveus, R., Egelberg, J. \& Selvig, K. A. (1978) The effect of topical citric acid application on the healing of experimental furcation defects in dogs. Journal of Periodontal Research 13, 538-549.

Hill, R. W., Ramfjord, S. P., Morrison, E. C., Appleberry, E. A., Caffesse, R. G., Kerry, G. J. \& Nissle, R. R. (1981) Four types of periodontal treatment compared over two years. Journal of Periodontology 52, 655-662.

Knowles, J. W., Burgett, F. G., Nissle, R. R., Shick, R. A., Morrison, E. C. \& Ramfjord, S. P. (1979) Results of periodontal treatment related to pocket depth and attachment level. 8 years. Journal of Periodontology 50, 225-233.

Loe, H. \& Silness, J. (1963) Periodontal disease in pregnancy. I. Prevalence and severity. Acta Odontologica Scandinavica 21, 533-551.

Nilveus, R., Bogel, G., Crigger, M., Egelberg, J. \& Selvig, K. A. (1980) The effect of topical citric acid application on the healing of experimental furcation defects in dogs. II. Healing after repeated surgery. Journal of Periodontal Research 15, 544-550. 
ment de fluide gingival, indice de plaque, atteinte des furcations, niveau de l'attache et profondeur de sondage. Une analyse statistique de toutes les données a été faite en utilisant le test- $t$ pour observations appariées, le test du $\chi^{2}$ et le test de probabilité exacte de Fisher.

Les résultats biométriques montraient qu'on obtenait avec les 2 méthodes une perte de l'attache dans les poches peu profondes et un gain dans les poches profondes. A 6 mois, les 2 méthodes donnaient les mêmes niveaux de récession et de réduction de la profondeur de sondage. L'indice gingival, l'indice de plaque et les valeurs pour les furcations étaient réduits à 3 mois et à 6 mois après l'intervention. Les valeurs de l'écoulement de fluide gingival restaient dans l'ensemble inchangées jusqu'à 6 mois après l'intervention.
Nilveus, R. \& Egelberg, J. (1980) The effect of topical citric application on the healing of experimental furcation defects in dogs. Journal of Periodontal Research 15, 551-560.

Nyman, S., Rosling, B. \& Lindhe, J. (1975) Effect of professional tooth cleaning on healing after periodontal surgery. Journal of Clinical Periodontology 2, 80-86.

Ramfjord, S. P. (1967) The periodontal disease index (PDI). Journal of Periodontology 38, 602-611.

Ramfjord, S. P., Knowles, J. W., Nissle, R. R., Burgett, F. G. \& Shick, R. A. (1975) Results following three modalities of periotherapy. Journal of Periodontology 46, 522-526.

Register, A. A. \& Burdick, F. (1975) Accelerated reattachment with cementogenesis to dentin, demineralization "in situ". I. Optimum range. Journal of Periodontology 46, 646-655.

Renvert, S. \& Egelberg, J. (1981) Healing after treatment of periodontal intraosseous defect. II. Effect of citric acid conditioning of the root surface. Journal of Clinical Periodontology 8, 459-473.

Rosling, B., Nyman, S., Lindhe, J. \& Jern, B. (1976) The healing potential of the periodontal tissues following different techniques of periodontal surgery in plaque-free dentitions. A 2 year clinical study. Journal of Clinical Periodontology 3, 233-250.

Silness, J. \& Loe, H. (1964) Periodontal disease in pregnancy. II. Correlation between oral hygiene and periodontal condition. Acta Odontologica Scandinavica 22, 121-135.

Stahl, S. S. \& Froum, S. J. (1977) Human clinical and histologic repair responses following the use of citric acid and perio therapy. Journal of Periodontology 48, 261-266.

Address:

Dr. B. A. Smith

Department of Periodontics

The University of Michigan

School of Dentistry

Ann Arbor, Michigan 48109

USA 
This document is a scanned copy of a printed document. No warranty is given about the accuracy of the copy. Users should refer to the original published version of the material. 